\title{
Removal of organic contaminants from water by using the polypropylene-based monoliths \\ By
}

\section{Nadeem Baig*1,2, Tawfik A. Saleh ${ }^{1}$}

${ }^{1}$ Chemistry Department, King Fahd University of Petroleum and Minerals, Dhahran 31261, Saudi Arabia.

${ }^{2}$ Center for Research Excellence in Desalination \& Water Treatment, King Fahd University of Petroleum and Minerals, Dhahran 31261, Saudi Arabia Email: nadeembaig@kfupm.edu.sa; nadeembaig38@gmail.com

\section{Introduction}

Organic contaminates in water are the major contributor in water pollution

The oil related organic contaminants in water are being added by food industries, textile, petrochemical and periodical oil spills incidents. ${ }^{1}$

The organic contaminants are continuously increasing due to rapid industrialization, urbanization and high energy demands from conventional sources

To get rid of oil and oil related organic contaminants from water various conventional methods are used such as skimmers, solidifiers, bioremediation, and dispersants.

> Some of these methods are suffering from high cost, poor separation efficiency and can become a source of addition of secondary pollutants. ${ }^{2}$

There is a need to develop new material that display specificity towards the organic pollutants in water

The hydrophobic and oleophilic porous surfaces can be proved impressive for the selective separation of the oil-related contaminants from the water.

\section{Synthesis of Magnetic Monolith of $\mathbf{P P}^{3}$}

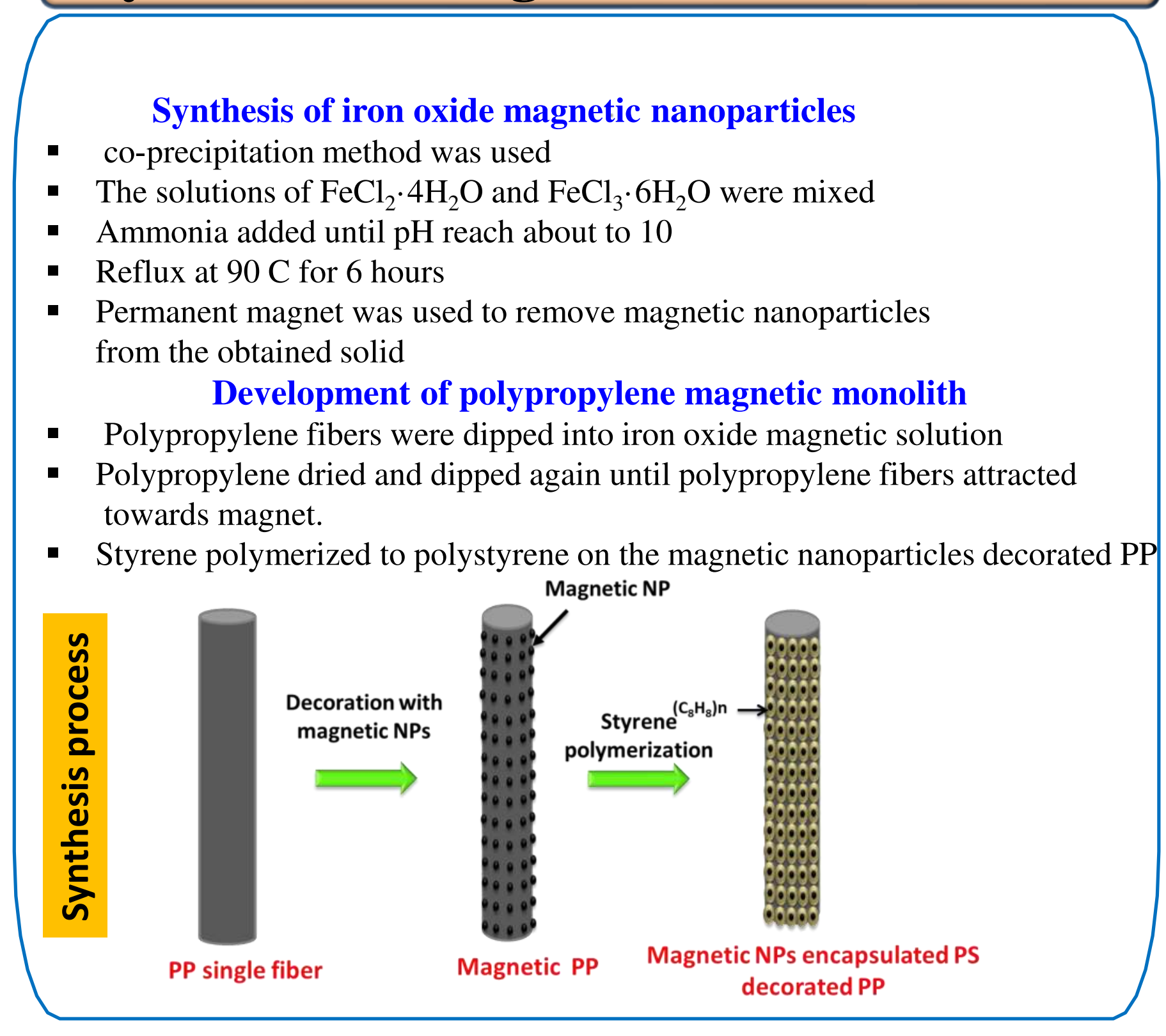

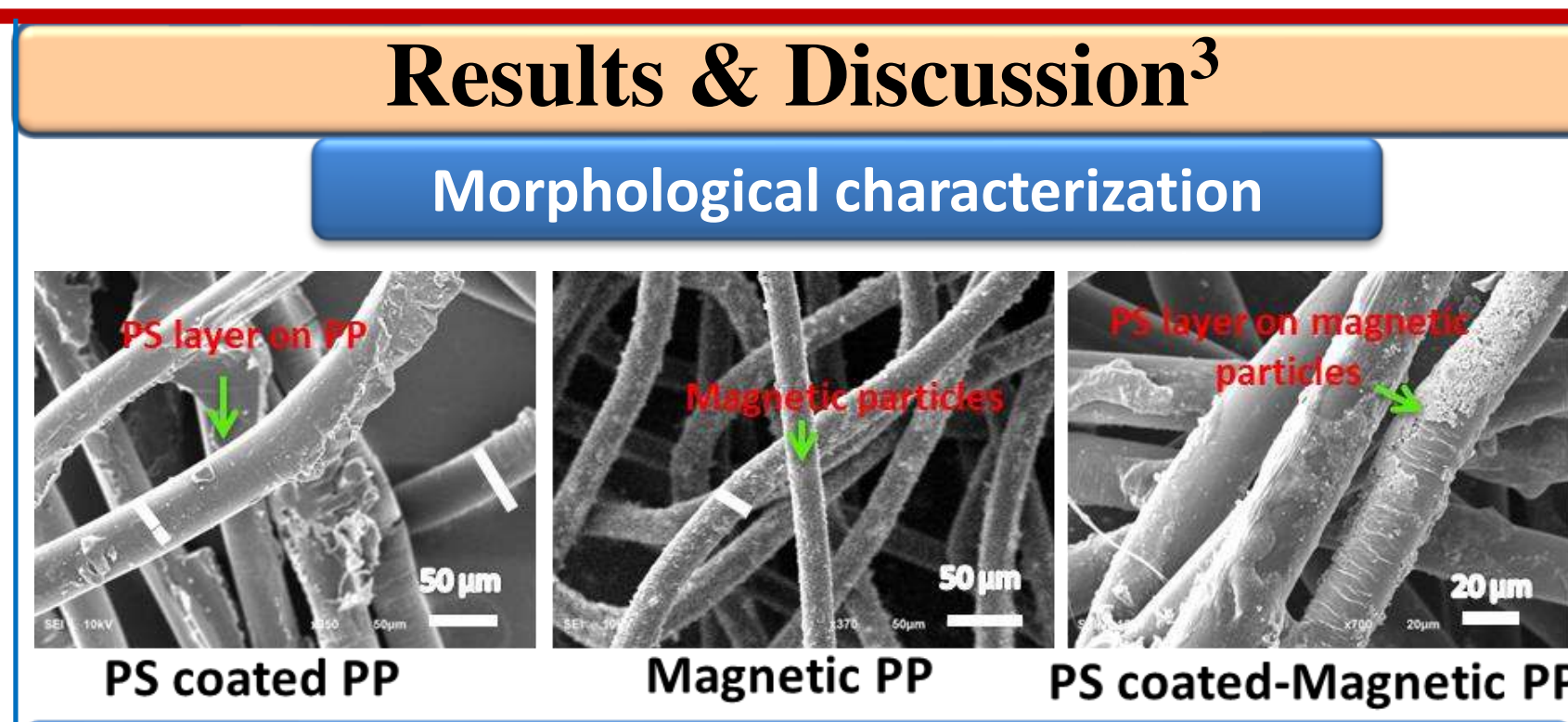

EDX and FTIR Spectra
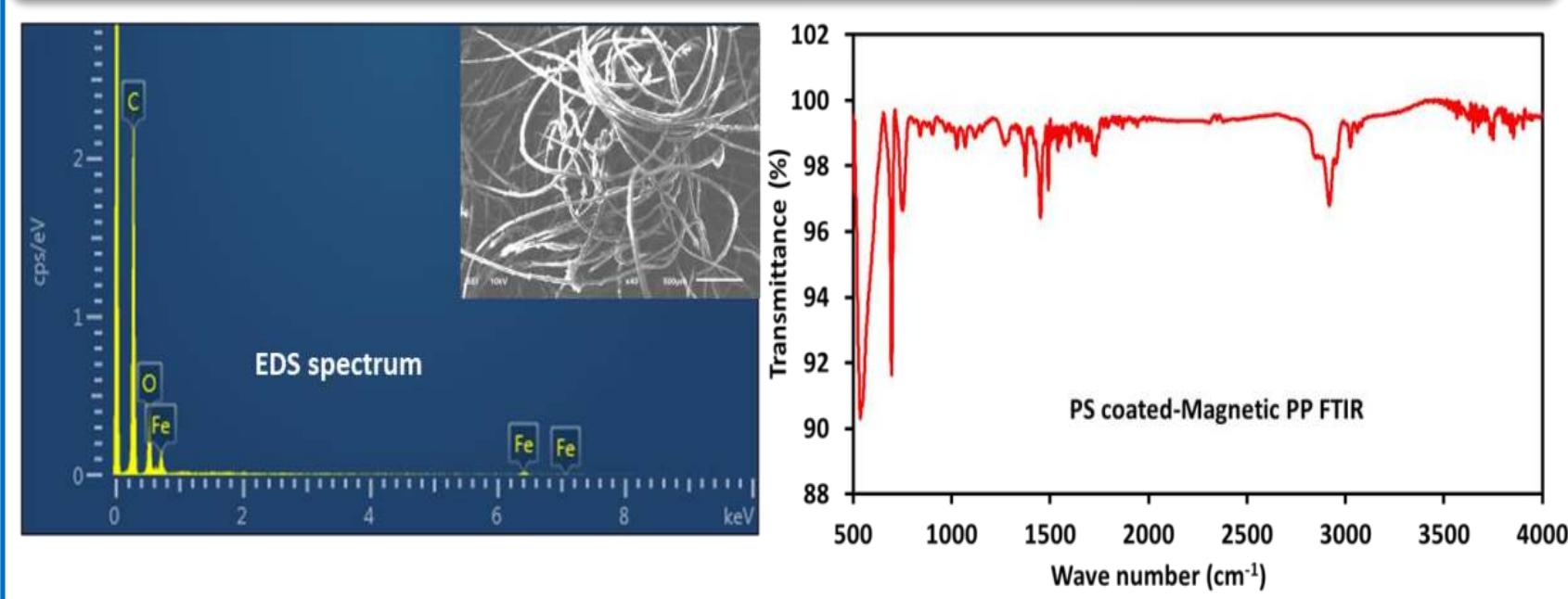

Magnetic behavior and water CA

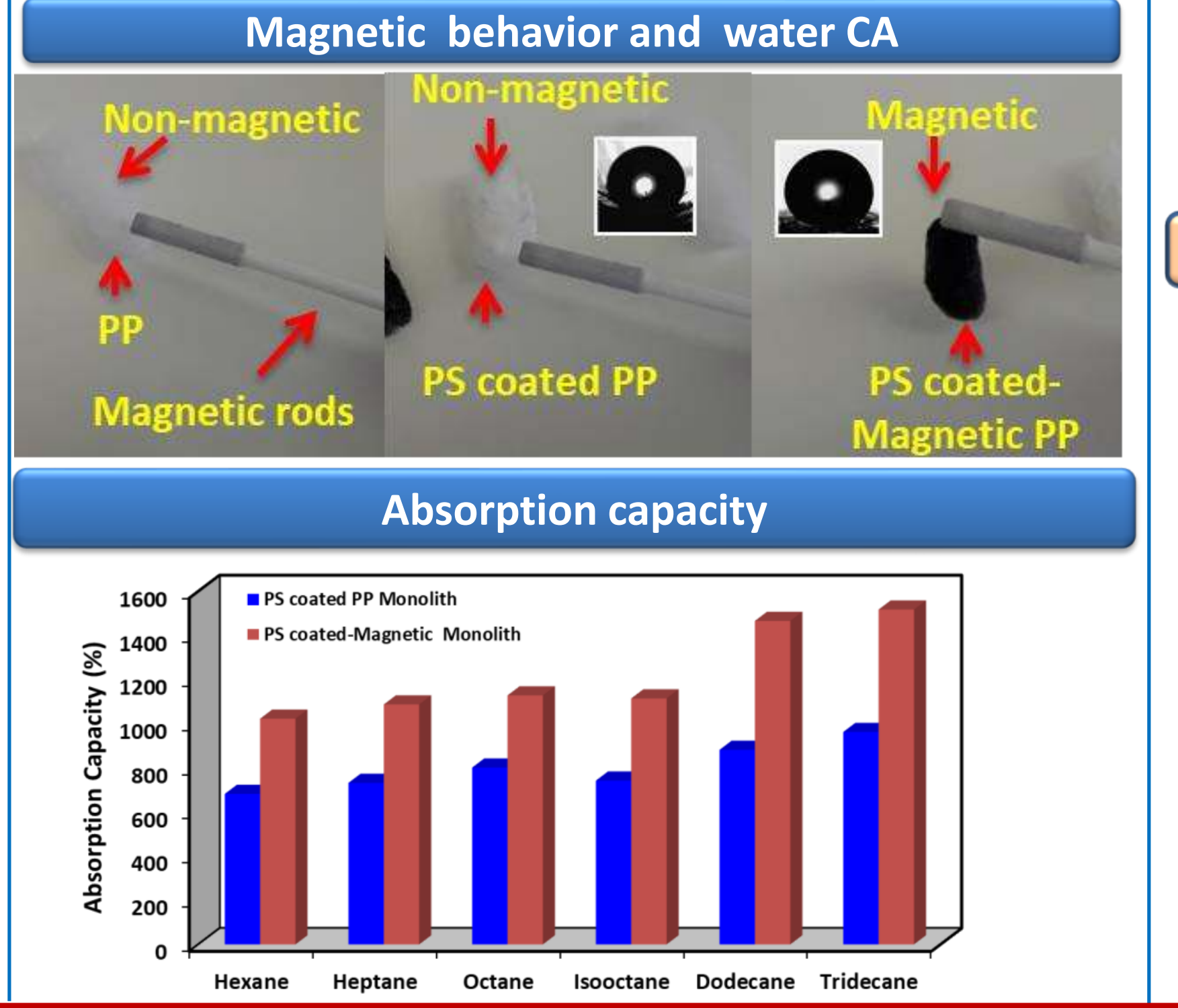

Separation of organic contaminants from water

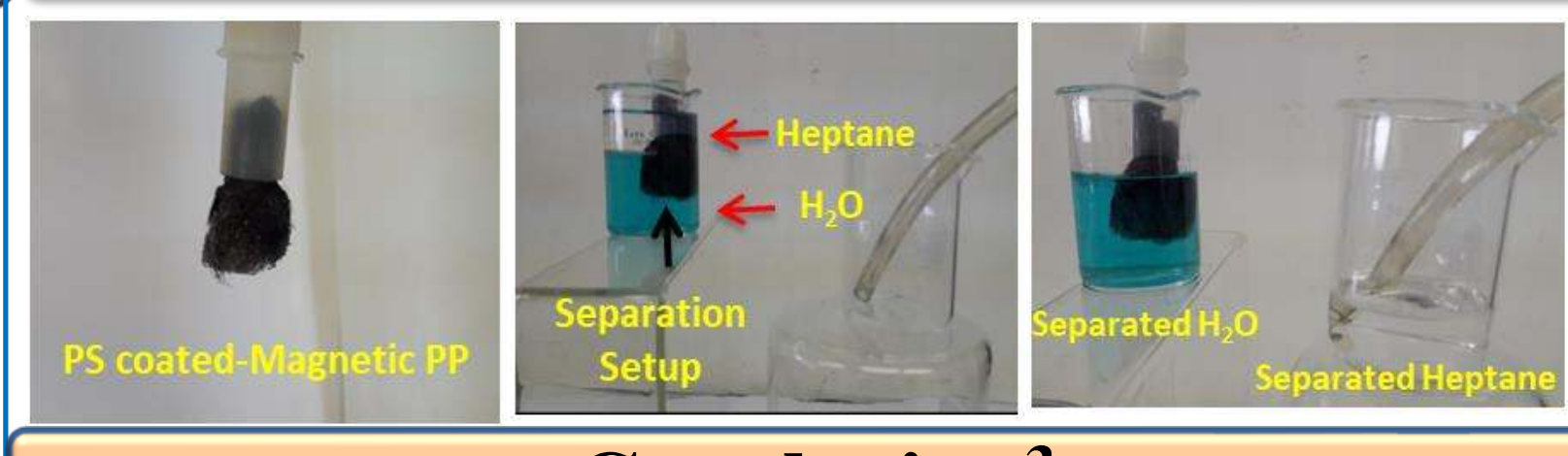

\section{Conclusion $^{3}$}

- Introduction of magnetic nanomaterial improved the surface area of the monolith to $221 \mathrm{~m}^{2} / \mathrm{g}$.

- PS coated Magnetic PP was found magnetically active

* Developed PS coated Magnetic PP has exhibited good

Dobicity with water contact angle of

- PS coated Magnetic PP can be used for the spot removal of the

organic contaminants from the water

* The absorption capacity of PS coated Magnetic PP was found in the range of $1023-1517 \%$

* PS coated Magnetic PP can be used for dynamic separation of non-polar organic contaminants

* During separation of the heptane and water the flux of 13365 $\mathrm{Lm}^{-2} \mathrm{~h}^{-1}$ was observed

- It can be used mutiple time for the separation of the non-polar organic contaminants from water

- Synthesis process is simple and cost-effective and can be used for large scale production

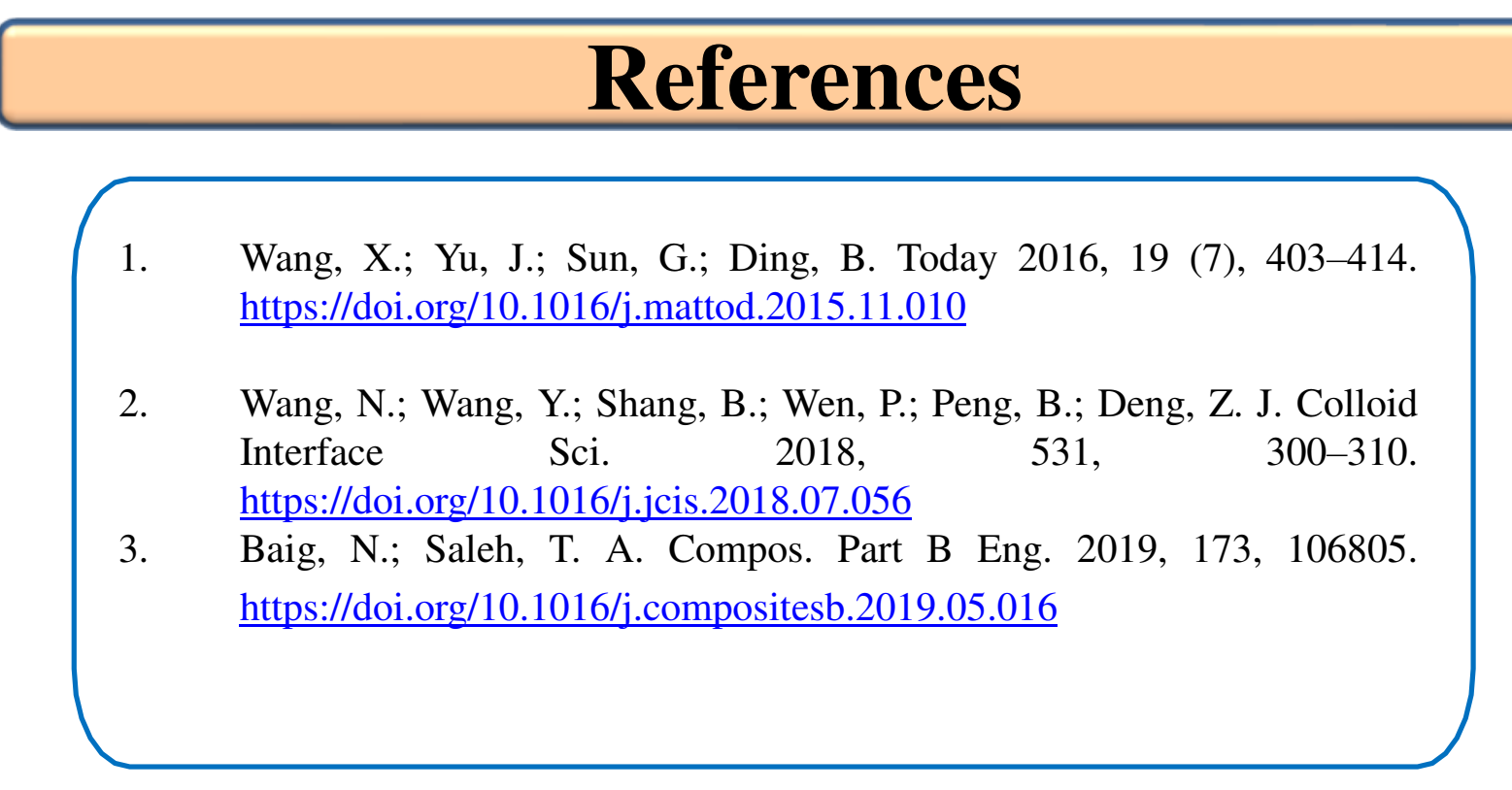

Acknowledgement: Authors gratefully acknowledge the support provided by the "King Fahd University of Petroleum and Minerals, Dhahran, Saudi Arabia". 